\title{
Current and future perspectives of digital microscopy with fluorescence confocal microscope for prostate tissue interpretation: a narrative review
}

\author{
Bernardo Rocco ${ }^{1}$, Alessia Cimadamore ${ }^{2}$, Luca Sarchi ${ }^{1}$, Luca Reggiani Bonetti ${ }^{3}$, Laura Bertoni ${ }^{4}$, \\ Paola Azzoni ${ }^{4}$, Simone Assumma ${ }^{1}$, Filippo Turri ${ }^{1}$, Giorgio Bozzini ${ }^{5}$, Ahmed Eissa ${ }^{6}$, Salvatore Micali ${ }^{1}$, \\ Giampaolo Bianchi ${ }^{1}$, Antonino Maiorana ${ }^{3}$, Rodolfo Montironi ${ }^{2}$, Giovanni Pellacani ${ }^{7}$, \\ Maria Chiara Sighinolfi ${ }^{1}$
}

${ }^{1}$ Department of Urology, University of Modena and Reggio Emilia, Modena, Italy; ${ }^{2}$ Department of Pathology, Polytechnic University of the Marche Region, Ancona, Italy; ${ }^{3}$ Department of Pathology, University of Modena and Reggio Emilia, Modena, Italy; ${ }^{4}$ Department of Human Anatomy, University of Modena and Reggio Emilia, Modena, Italy; ${ }^{5}$ ASST Valle Olona, Varese, Italy; ${ }^{\circ}$ Department of Urology, Faculty of Medicine, Tanta University, Tanta, Egypt; ${ }^{7}$ Department of Dermatology, University of Modena and Reggio Emilia, Modena, Italy

Contributions: (I) Conception and design: B Rocco, MC Sighinolfi; (II) Administrative support: B Rocco; (III) Provision of study materials or patients: B Rocco, MC Sighinolfi, L Sarchi, S Assumma; (IV) Collection and assembly of data: B Rocco, MC Sighinolfi, L Sarchi, S Assumma, A Eissa; (V) Data analysis and interpretation: B Rocco, MC Sighinolfi; (VI) Manuscript writing: All authors; (VII) Final approval of manuscript: All authors. Correspondence to: Maria Chiara Sighinolfi, MD, PhD. Department of Urology, University of Modena and Reggio Emilia, Via del Pozzo 71 , 41100 Modena, Italy. Email: sighinolfic@yahoo.com.

\begin{abstract}
Fluorescence confocal microscopy (FCM) is an optical imaging technique providing digital microscopical images of fresh tissue in a real time fashion, without conventional processing. FCM has been widely applied in several fields of dermatology, including the detection of basal cell carcinoma and of cutaneous inflammatory diseases. The aim of the paper is to provide an overview of FCM applications in the field of prostate tissue interpretation and prostate cancer (PCa) detection. A Literature search (PubMed \& Web of Science) was performed to identify articles concerned with the clinical and surgical applications of FCM in prostatic and periprostatic tissues interpretation. Overall, six articles were identified. All articles investigated the level of agreement between FCM and conventional histopathological analysis (hematoxylineosin, HE) for the discrimination between normal and PCa tissues. An investigative article on prostate samples retrieved from radical prostatectomy (RP) specimens and an atlas of FCM digital images from the same series were found. Two prospective clinical trials, comparing FCM and HE, pointed out a "substantial" to "almost perfect" discriminative performance of FCM for the diagnosis of PCa on prostate biopsy core. Finally, two studies investigated the intra-operative role of FCM during RP for the control of surgical dissection. In this setting, FCM could be used to analyse samples retrieved from suspicious peri-prostatic areas; FCM has also been tested for an en-face evaluation of flat slices obtained from the systematic sampling of the posterolateral aspects of the prostate, in a NeuroSAFE-like approach. Generally, FCM provides digital microscopical images of fresh tissue in a real time fashion, without requiring conventional processing. Currently, available studies confirmed a high concordance with conventional pathology for the detection of PCa. Further studies are required to validate the technology, to evaluate ISUP score attribution and to implement the fields of application of FCM for the treatment of prostate diseases.
\end{abstract}

Keywords: Prostate cancer; diagnosis; fluorescence confocal microscopy; digitalization

Submitted Sep 07, 2020. Accepted for publication Dec 30, 2020.

doi: $10.21037 /$ tau-20-1237

View this article at: http://dx.doi.org/10.21037/tau-20-1237 


\section{Introduction}

Fluorescence confocal microscopy (FCM) represents an imaging technique that provides digital images of fresh tissue in a real time fashion, without conventional processing. Microscopical images are given in a hematoxylin-eosin (HE) like appearance, resembling traditional slides pathologists are accustomed to deal with. The FCM analysis on the fresh sample allows for the preservation of specimen integrity for subsequent histopathological examination and ancillary studies such as immunohistochemistry (1).

When compared with the conventional wide field light microscope, the confocal microscope is based on an improvement in both maximum lateral resolution $(0.25$ $v s .0 .5 \mu \mathrm{m})$ and maximum axial resolution $(0.7 v s .1 .6 \mu \mathrm{m})$. The ability of the device to eliminate the "out-of-focus" brightness at a range of magnifications represents a feature typical of FCM (2). Depending on the laser wavelength, reflectance $(488 \mathrm{~nm})$ and fluorescence $(785 \mathrm{~nm})$ can be applied; modern FCM devices can combine the two modalities to characterize either stromal patterns and subcellular details, providing simultaneously two color scale mosaics resembling HE. Fluorescence mode relies on the use of dyes, with Acridine Orange being the mostly used; it binds specifically to DNA allowing the clear visualization of the nuclei.

Ex vivo FCM has been applied in the field of dermatology (3-5) and has been tested in terms of agreement with conventional frozen sections analysis for the detection of basal cell carcinoma and cutaneous inflammatory diseases, resulting in high levels of accuracy for FCM from a prospective study (3). Similarly, when applied to the interpretation of lung, liver, adrenal gland, kidney, bone, pleura, lymph nodes, and soft tissues, it has displayed a $95.2 \%$ of agreement with conventional HE (6).

In the urological field, it has been successfully applied for the interpretation of renal tissue. Mir et al. reported $100 \%$ of agreement with HE for the recognition of both tumor tissue and normal renal parenchyma, in the setting of the renal biopsy procedure (7). The aim was to identify a proper core sample, and the real-time analysis from FCM turned out likely to reduce the amount of non-diagnostic renal mass biopsy (7).

The aim of the current review is to report the available evidences about FCM for the interpretation of prostate tissue and to define the state of the art for its application for prostate cancer $(\mathrm{PCa})$ diagnosis and treatment. We present the following article in accordance with the Narrative Review reporting checklist (available at http://dx.doi. org/10.21037/tau-20-1237).

\section{Methods}

This narrative review was performed by the Department of Urology in the University of Modena \& Reggio Emilia, Italy, where the FCM technology has been used by dermatologists and other specialties for years. In October 2020, a comprehensive search of the literature was performed to identify the records discussing the clinical and surgical applications of FCM in prostatic and periprostatic tissues interpretation. Two authors (MCS \& LS) searched the PubMed and Web of Science databases using a combination of the following keywords; "prostate cancer", "radical prostatectomy", "prostate", "Digital frozen section", "fluorescence confocal microscopy", "fluorescence confocal microscope", and "fluorescent confocal microscopy". Several search filters were used during the search including the language and the time filters to include all the types of articles published in English language within the last 10 years. Generally, the search revealed 156 records. After the removal of duplicates, the records were screened by the title and abstract to identify all the articles concerned with the use of FCM in the examination of prostatic tissues. Overall, six clinical studies were identified $(1,8-12)$. Other articles dealing with historical aspects and technological background of confocal microscopy (13-16) were additionally discussed.

In this setting, the current review focuses particularly on the evaluation of prostate specimen both in prostate biopsy and radical prostatectomy surgical margin evaluation and explore the following topics:

* Historical background of clinical interest;

* Description of the fluorescence confocal microscope (device);

* Microscopical interpretation of prostate tissue with FCM;

* Current clinical applications of FCM (for prostate cancer diagnosis, during surgical treatment);

* Learning curve;

* Limitations.

\section{Results}

All the included records investigated the level of agreement between FCM and conventional histopathological analysis 
(HE) for the discrimination between normal and $\mathrm{PCa}$ tissue (1,8-12), of which, an investigative article on prostate samples retrieved from RP specimens and an atlas of FCM digital images discussed the same series of patients $(1,8)$, and two prospective clinical trials, compared FCM and HE in the setting of prostate biopsy, to obtain microscopical diagnosis of PCa $(10,11)$. Finally, two studies investigated the intra-operative role of FCM during RP for the control of surgical dissection $(9,12)$.

\section{Historical background of FCM technology}

The first confocal microscope was developed by Marvin Minsky in 1955 (13). The first version was a transmitted light microscope, consisting of a light source with a small hole in front of it through which a beam of light passed, then focused by a lens on the biological sample (15). Once through the biological sample, the beam of light was then focused by a second lens and a second small hole with the same focus as the first; basically, this is the reason of "confocal" definition. The light that passed through the second hole impacted against a detector producing a signal that is proportional to the brightness of the beam that passed through the hole, in addition the second hole shielded the light above and below the plane. The image was constructed by manually moving the sample $(2,15)$.

Another version of the same microscope was the reflected light microscope: it still consisted of a light source and a small hole producing a light beam: the light was reflected by a dichromatic mirror and focused thanks to a lens on the biological sample. The light was then reflected by the sample and then focused by the same lens as before, passing through the same dichromatic mirror and through a second hole impacting on the detector $(2,15,16)$.

Since these first prototypes, there have been many advances in technology including laser-based light sources, optimized reflective mirrors and filters, more efficient methods to obtain data, more efficient and silent photodetectors, improved biological sample preparation, faster computers, software to analyze images, high resolution displays, innovative bioinformatics methods $(2,15,16)$.

In the late 1980s, a group of researchers in Cambridge built a confocal laser microscope that could be considered the final prototype of recent ones used for research and clinical purposes. This microscope produced optical sections thin enough to analyze the elements of interest; thickness could be adjusted by changing the diameter of the hole in front of the detector, allowing for image zooming without losing the resolution, by decreasing the region of the sample scanned. Modern confocal microscopes could be classified according to the scanning method of the biological sample, that could consist either of the movement of the sample (stage scanning) or of the movement of the light beam (beam scanning) $(2,15,16)$.

Further progress occurred during the very recent decades-involving the stability and power of the laser (decreased in the latter microscopes to avoid photo damage), the effectiveness of dichromatic mirrors and photo detectors, electronic filters and finally implying improvement in software systems for digitalization. These advances make confocal microscopes more versatile for a number of current applications.

\section{Current fluorescence confocal device available for prostate clinical application}

The fluorescence confocal microscopy (FCM) with VivaScope ${ }^{\circledR} 2500$ M-G4 (Mavig GmbH, Munich, Germany; Caliber I.D.; Rochester NY, USA) is an optical imaging tool that works with two different lasers, reflectance $(785 \mathrm{~nm})$ and fluorescence $(488 \mathrm{~nm})$ modes $(1,14)$. In the prostate setting, FCM has been used for the real time interpretation of fresh dissected specimens (ex vivo). FCM has maximum examination depth of $200 \mu \mathrm{m}$, a vertical resolution of up to $4 \mu \mathrm{m}$, a magnification of $\times 550$ and a maximum scan size of $25 \times 25 \mathrm{~mm}$. Remarkably, the penetration depth can be increased by modifying the laser power and/or the incubation period of the specimen into the dye. The reconstructed image is a collection of mosaic images (square-shaped images of $1024 \times 1024$ pixels). The laser filter has a $\times 38,0.85$ numerical aperture water immersion objective lens. The software packages VivaScan ${ }^{\circledR}$ (Version 11.0.1140 Mavig GmbH; Caliber I.D), VivaBlock ${ }^{\circledR}$, and VivaStack $^{\circledR}$ enable respectively the reconstruction of the images from the probes, the acquisition of multiple images in the $\mathrm{X} / \mathrm{Y}$ directions within a single plane at a fixed depth, and a survey of multiple frames along the $Z$ axis, visualizing deeper tissue $(1,14)$. The grayscale fluorescence and reflectance mosaics were digitally stained with color and combined. To make images similar to hematoxylin and eosin (HE) and to increase pathologists' confidence with their interpretation, the purple and pink colors were used to improve association with cellular nuclei and noncellular structures, respectively (14). Zoom capabilities enable an enhanced visualization of cell morphology details. 
The current device is small and compact and can be easily located in the operative theatre or in an office.

\section{Handling and preparation of specimens}

The fresh tissue-without formalin fixation-is separately stained in acridine orange dye, after a rapid rinse into saline. Acridine orange (Sigma- Aldrich $\left.{ }^{\circledR}\right), 0.6 \mathrm{mM}$ solution is the dye currently used in the clinical practice; sample is immersed for 30 seconds, then washed in saline solution. The so stained sample is then placed between two glass slides sealed with silicon glue and then positioned onto the FCM stage for image acquisition $(1,8)$. Both sides of the glass slides can be examined by the FCM, allowing deeper examination of the specimen. The whole process for each specimen-including staining and image acquisition-is completed within a maximum of $5 \mathrm{~min}$, mostly depending on sample size: the larger is the area to scan, the longer is the time required for image acquisition. Afterwards, the sample undergoes the conventional processing, including formalin fixation (immersion in 10\% neutral buffered formalin for 24-h) and paraffin embedded. When appropriate, the sample is managed to maintain the proper orientation during either FCM and HE histopathological examination $(1,12)$. The same process has proven to fit FCM examination of several urological tissues, including renal parenchyma (7), nodes and urothelium (unpresented data, manuscript in submission).

\section{Interpretation of prostate tissue}

Benign prostatic tissue and benign prostatic hyperplasia Benign prostate tissue is displayed as round-oval regular glands composed of the typical two cell layers consisting of an outer layer of low flat or cuboidal basal cells and an inner tall columnar mucin-secreting epithelial cells $(1,8,11)$. The glands are arranged in a non-infiltrating pattern of growth and are surrounded by a thin connective tissue. Corpora amylacea are well identifiable even if small dimension $(1,8,11)$. Hyperplastic changes (benign prostatic hyperplasia) are visible as the increase of the number of the epithelial (epithelial hyperplasia) pale to clear cytoplasm and enlarged nuclei without atypia and/or stromal cells (stromal hyperplasia). Stromal hyperplasia is represented by fibrovascular and fibromuscolar proliferation in which is possible to recognize spindle cells and hyalinized matrix. Hyperplastic glands at FCM clearly exhibit basal cells (Figure 1).

Glands with stratified epithelium, cells with pleomorphic nuclei and prominent nucleoli identify high grade prostatic intraepithelial neoplasia in FCM images; basal cell layer is maintained $(1,8,11)$.

\section{Acinar adenocarcinoma}

Acinar adenocarcinoma is composed by closely packed irregular glands varying in size and shape, at time with a back-to-back distribution $(1,8,11)$. Small amount of stroma is interposed through the atypical glands and in some cases the glands are crowded with each other. In FCM, enlarged, hyperchromatic nuclei and prominent nucleolus are visible (purple) and represent a key-feature for the definition of the malignant morphology of the glands, associated with absence of basal cell layer and disarranged architecture of the glands (Figure 1).

The presence of cribriform glands is seemingly recognizable too, enabling the characterization of GG2 and GG3 tumors. When patterns of undifferentiated cell and poorly formed/fused glands are detected, PCa is graded as GG4 $(1,8,11)$.

\section{Interpretation of peri-prostatic soft tissue}

Samples from extra-prostatic soft tissues have been analyzed with regards to the discrimination between normal features-such as fatty, connective, muscular tissues-and the presence of persisting prostate cancer at this site, in case of extracapsular extension or positive surgical margin $(1,9)$. A study on 41 specimens from 20 patients described the FCM appearance of extra-prostatic components, as fatty tissues and muscle bundles, typically shown in a pinkcolour resembling eosin staining from the reflectance mode; opposite, prostate glandular parenchyma is different and displays a predominant purple colour provided by acridinestained nuclei and given from the fluorescence modality (Figure 2) (1,9). Rocco et al. (9), pointed out a level of agreement between FCM and HE that was $97.14 \%$ for muscular, $97.14 \%$ for nervous, $97.14 \%$ for vascular and $94.2 \%$ for fatty tissue. The detection of prostate glands, either benign and malignant, was feasible and concordant between FCM and the gold standard (Figure 2).

\section{Current clinical applications}

\section{Digital biopsy for the diagnosis of PCa}

Microscopical analysis is the key for PCa diagnosis and is achieved from prostate biopsy cores. Due to the high prevalence of $\mathrm{PCa}$ among adult males, prostate biopsy represents one of the most common procedures in 
A

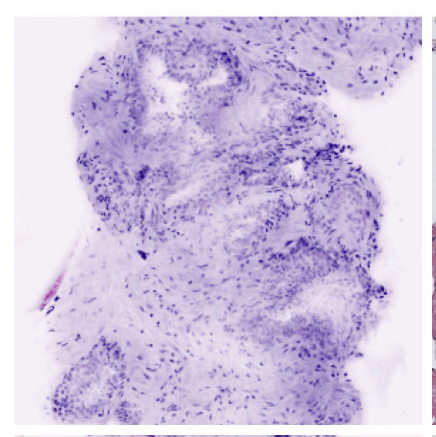

B

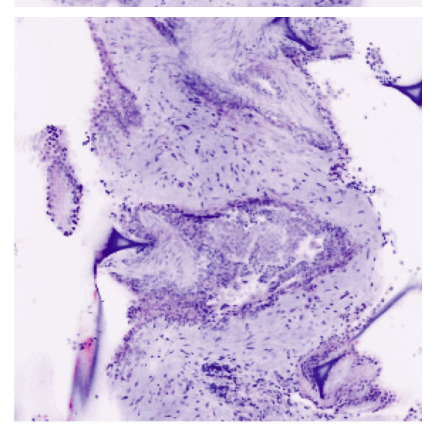

C
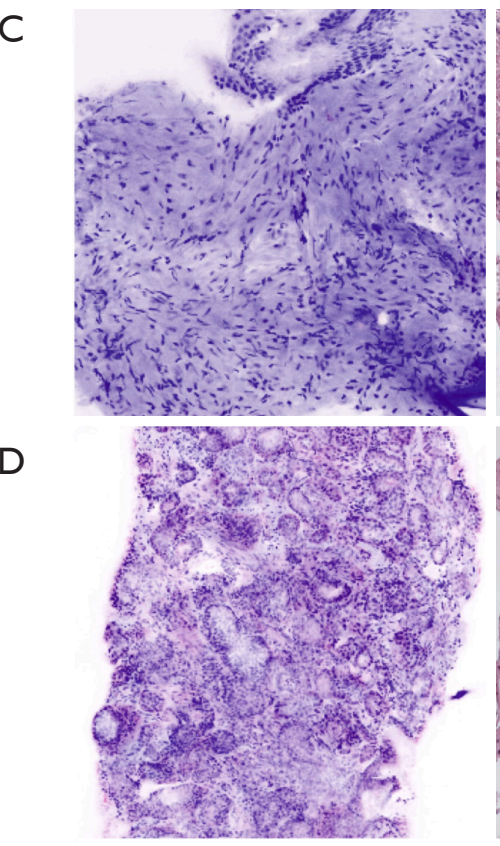
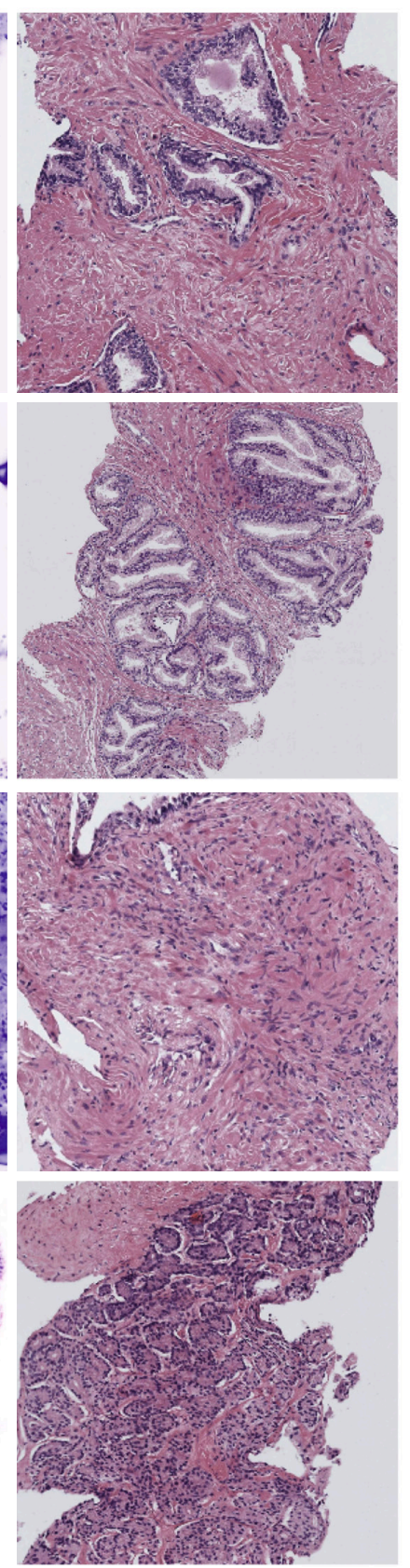

Figure 1 Images of prostate tissue in FCM (left) and HE (right). (A) Healthy tissue; (B) prostatic hyperplasia; (C) stromal hyperplasia; (D) prostate cancer (ISUP 3). Magnification: 10x.

urological practice; it is estimated that more than one million prostate biopsies are performed each year in Europe and the USA (17). Prostate biopsy can be performed with a systematic (12 cores from peripheral zone) or with a targeted sampling, on regions of interest (ROI) detected at mpMRI (18). A sampling involving either the targeted and a systematic approach seem to be those providing the best detection rate of clinically significant PCa (18). Once obtained the biopsy cores according to the approach, the conventional histopathological examination consists of processing, slide preparation and pathological reporting. Overall, it has been estimated that the mean turnaround 


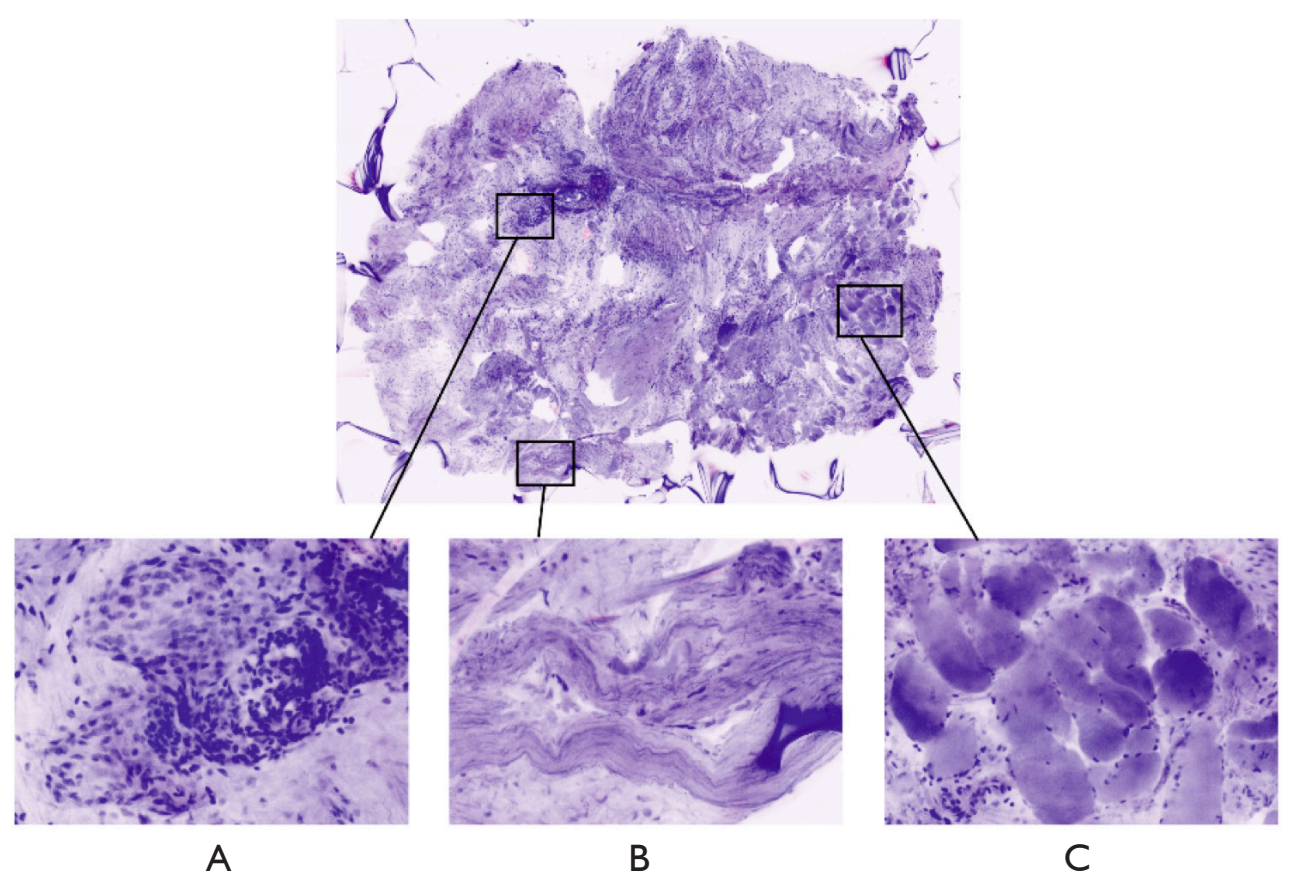

Figure 2 Depiction of a posterolateral surgical margin in FCM with components: (A) glandular tissue, (B) nerve, (C) muscular tissue. Magnification: $10 \times$.

time for reporting exceeds the one suggested from the College of American Pathologists (CAP) standard in a quarter of cases (19); this occurrence can be due to the time required for the preparation of the specimen-setup for formalin fixation, paraffin embedding, specimen cut and slide arrangement-and to the organizational burden often affecting several pathological departments in different geographical areas.

Two prospective clinical studies evaluated the ability of FCM to interpret in a real time fashion the prostate tissue on prostate biopsy cores $(10,11)$. As for dermatological studies, the primary endpoint was to evaluate the agreement between FCM and HE for prostate cancer diagnosis (presence $v s$. absence of $\mathrm{PCa}$ ). Rocco et al. (11), designed a prospective study that included 427 cores from either mpMRI targeted and random prostate areas from 54 patients. Biopsy cores were acquired digitally with FCM and then conventionally with HE analysis. All images-from digital biopsy and the corresponding HE digitized slideswere web-displayed to four pathologists from different institutions and with diverse backgrounds, in a random fashion. The outcome measure was the agreement between FCM and HE in terms of cancer diagnosis ( $\mathrm{k}$ value, AUC); the inter-observer agreement, between pathologists, has been evaluated as well. Overall, the diagnostic agreement between FCM and HE for the detection of cancerous tissue was almost perfect ( $\kappa=0.84$; range: 0.81 to 0.88 for 4 pathologists), with a $95.1 \%$ of correct diagnoses obtained (range 93.9-96.2\%). The discriminative performance of FCM was excellent, with an overall AUC of 0.92 (95\% CI: 0.90-0.94) (11).

Similarly, Marenco et al. (10), performed a prospective study on targeted prostate biopsy. A total of 182 cores from 65 ROI at mpMRI were taken and analyzed with FCM by a single pathologist. Then, HE analysis was performed and HE images were interpreted by a second pathologist, blinded to FCM results. The whole procedure is similar to that described by Rocco et al, except for the inclusion of both a "per-core" and a "per-ROI" analysis (10). Authors found an agreement between FCM and HE of 0.81 and 0.69 at biopsy core and ROI level, with high positive predictive value $(85 \%$ vs. $83.78 \%)$ and negative predictive value $(95.1 \%$ vs. $85.71 \%$ ) too, respectively.

With regard to ISUP scoring attribution, Marenco et al. (10), did not evaluate the issue arguing a lack of expertise to confidently assign grading to the FCM cores, thus invoking a sort of learning curve effect. Oppositely, Rocco et al. aimed to address ISUP scoring as a secondary endpoint, in terms of inter-observer agreement. Regrettably, the high inter-observer variability in the gold standard HE pathway 
made FCM findings inconclusive too (11); further studies on FCM ability to discriminate between ISUP groupsdesigned and powered to this outcome-are required.

\section{Digital biopsy during radical prostatectomy}

$\mathrm{RP}$ represents the gold standard surgical treatment for low and intermediate risk $\mathrm{PCa}$ and is part of the multimodal approach for high-risk and locally advanced disease (18). The goal of RP is the complete removal of the prostate while preserving potency and continence whenever possible. As stated from Walsh's preliminary study, the preservation of neurovascular bundles (NVB) is essential to erectile recovery but likely to impair surgical margin status (20). Positive surgical margins are found in $15 \%$ of cases when not stratified by T stage; for pT2 the overall PSM rate is $9 \%$, whereas for a pT3 status is $37 \%$. Positive surgical margins may affect biochemical recurrence and finally PCa specific mortality (21-23).

In the trade-off between the preservation of nerves and the risk of positive SM, the intra-operative microscopical control of surgical dissection may be the cornerstone. Some studies on confocal laser endomicroscopy with Cellvizio attempted to check in vivo the location of NVBs in the effort to spare as much as possible healthy tissue: however, either an experimental (24) and a clinical in vivo study (23) reported poor outcomes due to lack of depth, small FOV and, mostly, to a difficult interpretation of black-and-white images from Cellvizio that limit a comprehensive evaluation of the prostate capsule (25).

Nowadays, the microscopical assessment of surgical margin on the fresh specimen (ex vivo) is the most common way to control surgical dissection in a real time fashion. Frozen section analysis (FS) of the prostate represents the standard approach to this purpose (18). However, the ideal sampling method (26) and the actual role of FS in reducing positive SM are still debated $(27,28)$.

Nevertheless, the study from Schlomm et al. (29), demonstrated that when applied systematically to the posterolateral margin of the prostate, FS at this site may improve the rate of NS procedures while preserving a negative margin status. Remarkably, the NeuroSAFE approach as described by the authors requires a dedicated setup, with a fully equipped laboratory including cryostats, technicians handling and preparing the specimen, pathologists on-site ready to interpret it and to provide the surgeon an immediate evaluation of the specimen (30). Overall, the NeuroSAFE requires 35 minutes in facilities with an appropriate setup, thus being not affordable in all pathological departments (30).

In this scenery, the role of FCM digital biopsy has been described to implement the real time control of surgical dissection. Microscopical evaluation with FCM was proven to fit either a random or a systematic sampling. During prostate dissection, suspect areas could be detected and would prompt a wider excision, if not further characterized. This is the case of inflammatory reaction deriving from prostate biopsy, such as traumatic neuroma (31): surgeon's perception is that of a thigh adhesion of peri-prostatic tissue to the capsule and could be misinterpreted as an area of extracapsular extension (ECE), thus requiring a broader plane of dissection. Rocco et al. (9), addressed the issue with a prospective study on 41 samples retrieved during RALP at suspicious areas or from sites corresponding to suspicious ECE at pre-operative mpMRI. The endpoint was to describe the ability of FCM to detect the possible presence of prostate glands within extra-prostatic soft tissues, indicating ECE or an inadvertent incision inside the prostate capsule (9). To this purpose, the study evaluated the concordance between FCM and HE for the evaluation of each component of the extra-prostatic environment. As a result, the Authors found an agreement percentage exceeding $90 \%$ for all elements, with connective, fatty and muscular tissues being easily interpreted; within these tissues, the presence of prostatic glandular pattern is clearly distinguishable (9).

Beyond the analysis of small samples suspicious of cancer from the peri-prostatic areas, FCM has been applied for the en-face analysis of the prostatic surface, to assess for surgical margin status. As for the NeuroSAFE (29), the evaluation has been performed with a systematic sampling of the posterolateral aspect of the prostate, the area at risk of positive surgical margin during a nerve sparing procedure. In a prospective exploratory study, Rocco et al. (12), retrieved a slice shaved section at each lobe of the prostate specimen from 8 men undergoing radical prostatectomy (36 samples overall). Such slices were obtained with a tangential cut of the glands from the base to the apex and then flattened on the Vivascope ${ }^{\circledR}$ for an en-face visualization of the tissue by using the lowest thickness optical setup (12). Occasionally, longitudinal slices were divided into 2 or 3 parts to fit the $2.5 \mathrm{~cm} \times 2.5 \mathrm{~cm}$ scanning area, depending on prostate volume. The method differs from that performed with conventional frozen section (including NeuroSAFE), which relies on the circumferential analysis of the inked margin from a definite number of prostate transverse sections (10-12/lobe for NeuroSafe). In the study from 
Rocco et al. (12), images have been shared with two pathologists, and the study definitively confirmed a perfect concordance between FCM-applied to the flat specimenand conventional $\mathrm{HE}$ analysis applied to the circumferential margin of the prostate. The systematic acquisition of FCM digital margins has been performed within 25 minutes; only 1 out of 8 patients had a positive surgical margin in an area of ECE. In this case, since the Vivascope ${ }^{\circledR}$ is provided with a ruler, the exact location of neoplastic glands at the specimen's level was measured and tracked on the NVB, allowing for a focal secondary resection and preservation of healthy NVB tissue (12). Figure S1 displays the setup of the confocal microscope for prostate systematic sampling.

\section{Learning curve}

The learning curve effect for FCM interpretation of prostate tissue has been investigated by Bertoni et al. (1). Authors displayed 89 FCM digital images obtained from prostate specimen to two pathologists (a general and a dedicated uro-pathologist) blinded to the histopathologic diagnosis; pathologists were asked to report on the presence $v s$. absence of PCa. Then, after a time lapse of more than 90 days, they were asked to re-evaluate all the specimens, displayed in a novel random order. The agreement between the FCM and HE increased from a range $86-92 \%$ obtained from the first reading to $95 \%$ for both pathologists in the second reading. Noteworthy, $10 \%$ of specimens finally required immunohistochemistry for histopathological diagnosis, which may be responsible for the slight (5\%) disagreement obtained at the second round (1). Actually, the area under the receiver operating characteristic (ROC) curve at the first reading was 0.87 , thus indicating an excellent discriminative performance of FCM since the first reading, that is furtherly improved at the second one reaching AUC 0.92-0.93. Authors concluded that the learning curve for FCM is short due to HE-like appearance that makes easy the interpretation of prostate tissue (1).

Rocco et al. (11), did not address specifically the learning curve effect in their prospective study on prostate biopsy; however, the high level of agreement for prostate cancer detection from pathologists naïve to FCM may indirectly confirm a short learning curve effect for FCM reading, especially if considering that one of four pathologists was a resident at the time of the study.

It may be questionable if ISUP score attribution requires a longer learning curve (10), to make pathologists more confident with grade grouping. However, none of the cited studies was designed to this purpose, and further researches addressing specifically prostate cancer tissue are required.

\section{Limitations}

Despite the advantage of a straight acquisition of digital images, some technical features are not devoid of limitation. Firstly, the small scanning area fits the analysis of low sized specimen $(11,12)$, such as biopsies. The current commercially available device (MAVIG VivaScope ${ }^{\circledR} 2500$ ) allows to scan areas up to $2.5 \mathrm{~cm} \times 2.5 \mathrm{~cm}$ with a single field of view (FOV) of $500 \mu \mathrm{m} \times 500 \mu \mathrm{m}$. To obtain a mosaic image of $2.5 \mathrm{~cm} \times 2.5 \mathrm{~cm}$, the sample is moved under the objective, by means of a motorized stage and hundreds of FOVs are automatically stitched together by the microscope software during the acquisition process. To further enlarge the maximum scanning area, a motorized stage, allowing larger movement of the sample under the objective, could be implemented (14).

Secondly, the time to acquire the images is strictly dependent on specimen size $(11,12)$ : the smaller the sample, the faster the scanning is performed. Actually, the time of acquisition of a given area from a specimen is a function of the number of FOVs necessary to create a mosaic image of that area. An increase of the FOV would decrease the number of FOVs necessary to create the mosaic image and thus reduce specimen acquisition time.

Finally, there is room for implementation of storage programs and reporting software, to share images, to homogenize the reporting, to assist future researches with standardized methods to assess outcomes.

\section{Future perspectives}

\section{Clinical Perspectives}

The perspective of a real time diagnosis of PCa from digital biopsy opens unexplored fields of application. Recently, focal therapy has been proposed as an option for localized unilateral PCa. In this setting, patients could receive both a diagnostic and a therapeutic procedure during the same session: digital biopsy of cores from a mpMRI zone can be acquired with FCM and interpreted in real-time by a remote pathologist, and the diagnosis of $\mathrm{PCa}$ would prompt the concurrent treatment of the cancerous area $(10,11)$. Similarly, in case of bladder outlet dysfunction with mildly elevated PSA level, the risk of possible PCa could be ruled out with FCM performed at the same time of the planned treatment for BPH. In this setting, FCM is likely to avoid 
the need for a prior prostate biopsy, that would imply prolonged turnaround time and delay of the underlying condition.

With regard to the intra-operative setting, the perspective of a tailored surgical dissection is no longer a future development, but rather a current scenario whose implementation may lead to improvement in functional outcomes. From the proof of concept study of Rocco and coworkers (12), FCM allows the precise localization of the positive surgical margin on the posterolateral aspect of the prostate specimen, with the potential of tracking the same site on the spared NVB. Thus, the study poses the very basis for a tailored and focal secondary resection of NVB, without the need for a complete dissection. Actually, it has been demonstrated that only $23 \%$ to $42 \%$ of the secondarily resected NVB finally showed cancerous tissue (30): a partial wedge resection with FCM would be able to resect cancerous foci on NVB while sparing as much as possible healthy functional tissue. Though, further studies are required to evaluate the oncological benefit and the possible advantage in erectile function preservation.

Finally, the almost perfect discrimination between cancerous and normal tissue as described for the prostate could be transferred in diagnostic settings for different malignancies, potentially including all biopsy procedures performed for oncological suspicion.

\section{Organizational perspectives}

During the recent years, there has been a decrease in pathologist workforce in several health systems, including those from Canada and the UK (32). The inadequate staffing, occurring for $97 \%$ of the departments according to Metter et al., may possibly lead to diagnostic delays and increased costs, due to the need to rely on outsource services or temporary workers (32). The availability of straightly digitized FCM images is likely to simplify the pathway and it perfectly aligns with the current trend of digitalization, web-connection and real-time sharing. Digital microscopical images provide the advantage of remote accessibility and could be useful for a second opinion, for training purposes, for digital storage and are at the very basis of artificial intelligence and machine learning improvements (33-36). The health system is possibly enabled to provide partial services at different geographical sites at an efficient cost and to get the response from subspecialized and/or multiple experts, limiting the turnaround time. Actually, pathology is not devoid of the novel perspective of telemedicine: telepathology implies the transmission of microscopical images from one location to another for diagnostic purposes and could be useful in case of hospitals performing surgical procedures but lacking onsite pathology service, as occurs in low-income or developing countries (37-39). In this perspective, the implementation of FCM could have organizational effects fitting the double need of workload balancing and tele-reporting.

For these features, FCM may also apply to the current pandemic scenario. Besides, the handling and processing of specimen from Covid-19 infected patients is arising safety concerns in pathologists' community for the risk of pathogen viability through fresh tissue (40-43). A study on the detection of COVID-19 in different types of specimen revealed that faeces and blood could display SARS-CoV-2 RNA (41). The systematic use of frozen section analysis has been discouraged during the pandemic; thus, FCM opportunity to have specimens completely handled by the surgeon avoiding unnecessary exposure of additional personnel-technicians and pathologists—represents an advantage suitable for the pandemic scenery.

\section{Conclusion}

FCM has been tested in the setting of prostate tissue interpretation and displayed a diagnostic performance comparable to the one of conventional histopathology for the detection of cancer.

Summarizing, the advantages of FCM are:

- Straight acquisition of digital images from fresh specimen;

* Fast acquisition of digital images;

* Microscopical images provided in a HE-like appearance to which pathologists are confident;

* Very high concordance with conventional pathology;

* Preservation of tissue integrity for further histopathological analysis;

* Opportunity for real-time remote reporting, websharing and second opinion;

* No need for a steep learning curve for handling samples and for image interpretation;

* En-face analysis of a flattened sample, with the ability to maintain the proper orientation of the specimen and to precisely report the site of the malignant tissue inside the sample (measurement provided by FCM ruler).

FCM use in the field of prostate cancer is likely to increase in the near future; clinical trials with a multicentric 
fashion are invoked to confirm the transferability of the current evidence, to evaluate FCM reporting of ISUP scoring and to move forward toward novel surgical applications.

\section{Acknowledgments}

Funding: None.

\section{Footnote}

Provenance and Peer Review: This article was commissioned by the editorial office, Translational Andrology and Urology for the series "Update on Molecular Classification and Individualized Treatments of Genitourinary Tumors". The article has undergone external peer review.

Reporting Checklist: The authors have completed the NARRATIVE REVIEW reporting checklist. Available at http://dx.doi.org/10.21037/tau-20-1237

Conflicts of Interest: All authors have completed the ICMJE uniform disclosure form (available at http://dx.doi. org/10.21037/tau-20-1237). The series "Update on Molecular Classification and Individualized Treatments of Genitourinary Tumors" was commissioned by the editorial office without any funding or sponsorship. AC and RM served as the unpaid Guest Editors of the series. The authors have no other conflicts of interest to declare.

Ethical Statement: the authors are accountable for all aspects of the work in ensuring that questions related to the accuracy or integrity of any part of the work are appropriately investigated and resolved.

Open Access Statement: This is an Open Access article distributed in accordance with the Creative Commons Attribution-NonCommercial-NoDerivs 4.0 International License (CC BY-NC-ND 4.0), which permits the noncommercial replication and distribution of the article with the strict proviso that no changes or edits are made and the original work is properly cited (including links to both the formal publication through the relevant DOI and the license). See: https://creativecommons.org/licenses/by-nc-nd/4.0/.

\section{References}

1. Bertoni L, Puliatti S, Reggiani Bonetti L, et al. Ex vivo fluorescence confocal microscopy: prostatic and periprostatic tissues atlas and evaluation of the learning curve. Virchows Arch 2020;476:511-20.

2. Paddock SW, Eliceiri KW. Laser scanning confocal microscopy: History, applications, and related optical sectioning techniques. Methods Mol Biol 2014;1075:9-47.

3. Longo C, Pampena R, Bombonato C, et al. Diagnostic accuracy of ex vivo fluorescence confocal microscopy in Mohs surgery of basal cell carcinomas: a prospective study on 753 margins. Br J Dermatol 2019;180:1473-80.

4. Bertoni L, Azzoni P, Reggiani C, et al. Ex vivo fluorescence confocal microscopy for intraoperative, real-time diagnosis of cutaneous inflammatory diseases: A preliminary study. Exp Dermatol 2018;27:1152-9.

5. Reggiani C, Pellacani G, Reggiani Bonetti L, et al. An intraoperative study with ex vivo fluorescence confocal microscopy: diagnostic accuracy of the three visualization modalities. J Eur Acad Dermatol Venereol 2021;35:e92-4.

6. Krishnamurthy S, Sabir S, Ban K, et al. Comparison of Real-Time Fluorescence Confocal Digital Microscopy With Hematoxylin-Eosin-Stained Sections of CoreNeedle Biopsy Specimens. JAMA Netw Open 2020;3:e200476.

7. Mir MC, Bancalari B, Calatrava A, et al. Ex-vivo confocal fluorescence microscopy for rapid evaluation of renal core biopsy. Minerva Urol Nefrol 2020;72:109-13.

8. Puliatti S, Bertoni L, Pirola GM, et al. Ex vivo fluorescence confocal microscopy: the first application for real-time pathological examination of prostatic tissue. BJU Int 2019;124:469-76.

9. Rocco B, Sighinolfi MC, Bertoni L, et al. Realtime assessment of surgical margins during radical prostatectomy: a novel approach that uses fluorescence confocal microscopy for the evaluation of peri-prostatic soft tissue. BJU Int 2020;125:487-9.

10. Marenco J, Calatrava A, Casanova J, et al. Evaluation of Fluorescent Confocal Microscopy for Intraoperative Analysis of Prostate Biopsy Cores. Eur Urol Focus 2020. [Epub ahead of print]. doi: 10.1016/j.euf.2020.08.013.

11. Rocco B, Sighinolfi MC, Sandri M, et al. Digital Biopsy with Fluorescence Confocal Microscope for Effective Real-time Diagnosis of Prostate Cancer: A Prospective, Comparative Study. Eur Urol Oncol 2020. [Epub ahead of print]. doi: 10.1016/j.euo.2020.08.009.

12. Rocco B, Sighinolfi MC, Cimadamore A, et al. Digital frozen section of the prostate surface during radical prostatectomy: a novel approach to evaluate surgical margins. BJU Int 2020;126:336-8. 
13. Minsky M. Memoir on inventing the confocal scanning microscope. Scanning 1988;10:128-38.

14. MAVIG (2018) Datasheet VivaScope® 2500M-G4 [Internet]. [cited 2020 Nov 16]. Available online: https:// www.vivascope.de/wp-content/uploads/2019/06/DS_VS2500M-G4_287_0219-ohne-Mohs.pdf

15. Pawley JB. Handbook of biological confocal microscopy: Third edition. Handbook of Biological Confocal Microscopy: Third Edition. Springer US; 2006. 1-985 p.

16. Amos WB, White JG. How the confocal laser scanning microscope entered biological research. Biol Cell 2003;95:335-42.

17. Cicione A, De Nunzio C, Manno S, et al. An update on prostate biopsy in the era of magnetic resonance imaging. Minerva Urol Nefrol 2018;70:264-74.

18. European Association of Urology. EAU Guidelines: Prostate Cancer I Uroweb [Internet]. 2019. [cited 2020 Nov 16]. Available online: https://uroweb.org/guideline/ prostate-cancer/

19. Alshieban S, Al-Surimi K. Reducing turnaround time of surgical pathology reports in pathology and laboratory medicine departments. BMJ Qual Improv Reports 2015;4:u209223.w3773.

20. Walsh PC, Donker PJ. Impotence following radical prostatectomy: Insight into etiology and prevention. J Urol 1982;128:492-7.

21. Mauermann J, Fradet V, Lacombe L, et al. The impact of solitary and multiple positive surgical margins on hard clinical end points in 1712 adjuvant treatmentnaive p T2-4 N0 radical prostatectomy patients. Eur Urol 2013;64:19-25.

22. Yossepowitch O, Briganti A, Eastham JA, et al. Positive surgical margins after radical prostatectomy: A systematic review and contemporary update. Eur Urol 2014;65:303-13.

23. Lopez A, Zlatev DV, Mach KE, et al. Intraoperative Optical Biopsy during Robotic Assisted Radical Prostatectomy Using Confocal Endomicroscopy. J Urol 2016;195:1110-7.

24. Boyette LB, Reardon MA, Mirelman AJ, et al. Fiberoptic Imaging of Cavernous Nerves In Vivo. J Urol 2007;178:2694-700.

25. Jaulim A, Aydın A, Ebrahim F, et al. Imaging modalities aiding nerve-sparing during radical prostatectomy. Turk J Urol 2019;45:325-30.

26. Sighinolfi MC, Rocco B. Re: EAU Guidelines: Prostate Cancer 2019. Eur Urol 2019;76:871.

27. Dinneen EP, Van Der Slot M, Adasonla K, et al.
Intraoperative Frozen Section for Margin Evaluation During Radical Prostatectomy: A Systematic Review. Eur Urol Focus 2020;6:664-73.

28. Eissa A, Zoeir A, Sighinolfi MC, et al. "Real-time" Assessment of Surgical Margins During Radical Prostatectomy: State-of-the-Art. Clin Genitourin Cancer 2020;18:95-104.

29. Schlomm T, Tennstedt P, Huxhold C, et al. Neurovascular structure-adjacent frozen-section examination (NeuroSAFE) increases nerve-sparing frequency and reduces positive surgical margins in open and robot-assisted laparoscopic radical prostatectomy: Experience after 11069 consecutive patients. Eur Urol 2012;62:333-40.

30. Oxley J, Bray A, Rowe E. Could a Mohs technique make NeuroSAFE a viable option? BJU Int 2018;122:358-9.

31. Sighinolfi MC, Rocco B. Reply to Alessia Cimadamore, Marina Scarpelli, Liang Cheng, et al's Letter to the Editor, re: Maria Chiara Sighinolfi, Bernardo Rocco's Words of Wisdom re: EAU Guidelines: Prostate Cancer 2019. Mottet N, van den Bergh RCN, Briers E, et al. https:// uroweb. Eur Urol 2020;77:e128-9.

32. Metter DM, Colgan TJ, Leung ST, et al. Trends in the US and Canadian Pathologist Workforces From 2007 to 2017. JAMA Netw Open 2019;2:e194337.

33. Bulten W, Pinckaers H, van Boven H, et al. Automated deep-learning system for Gleason grading of prostate cancer using biopsies: a diagnostic study. Lancet Oncol 2020;21:233-41.

34. Ström P, Kartasalo K, Olsson H, et al. Artificial intelligence for diagnosis and grading of prostate cancer in biopsies: a population-based, diagnostic study. Lancet Oncol 2020;21:222-32.

35. Nir G, Karimi D, Goldenberg SL, et al. Comparison of Artificial Intelligence Techniques to Evaluate Performance of a Classifier for Automatic Grading of Prostate Cancer From Digitized Histopathologic Images. JAMA Netw Open 2019;2:e190442.

36. Chen PC, Gadepalli K, MacDonald R, et al. An augmented reality microscope with real-time artificial intelligence integration for cancer diagnosis. Nat Med 2019;25:1453-7.

37. Huang EY, Knight S, Guetter CR, et al. Telemedicine and telementoring in the surgical specialties: A narrative review. Am J Surg 2019;218:760-6.

38. Montironi R, Cheng L, Cimadamore A, et al. Uropathologists During the COVID-19 Pandemic: What Can Be Learned in Terms of Social Interaction, Visibility, and Social Distance. Eur Urol 2020;78:478-81. 
39. Cimadamore A, Lopez-Beltran A, Scarpelli M, et al. Digital pathology and COVID-19 and future crises: pathologists can safely diagnose cases from home using a consumer monitor and a mini PC. J Clin Pathol 2020;73:695-6.

40. Compérat E. What Does COVID-19 Mean for the Pathology-Urology Interaction? Eur Urol 2020;78:e43-4.

41. Wang W, Xu Y, Gao R, et al. Detection of SARSCoV-2 in Different Types of Clinical Specimens. JAMA

Cite this article as: Rocco B, Cimadamore A, Sarchi L, Bonetti LR, Bertoni L, Azzoni P, Assumma S, Turri F, Bozzini G, Eissa A, Micali S, Bianchi G, Maiorana A, Montironi R, Pellacani G, Sighinolfi MC. Current and future perspectives of digital microscopy with fluorescence confocal microscope for prostate tissue interpretation: a narrative review. Transl Androl Urol 2021;10(3):1569-1580. doi: 10.21037/tau-20-1237
2020;323:1843-4.

42. Gerston KF, Blumberg L, Tshabalala VA, et al. Viability of mycobacteria in formalin-fixed lungs. Hum Pathol 2004;35:571-5.

43. Badia JM, Rubio-Pérez I, Arias Díaz J, et al. Protocolo de actuación quirúrgica en casos confirmados o sospechosos de enfermedad por Ébola y otras enfermedades víricas altamente transmisibles. Cir Esp 2016;94:11-5. 


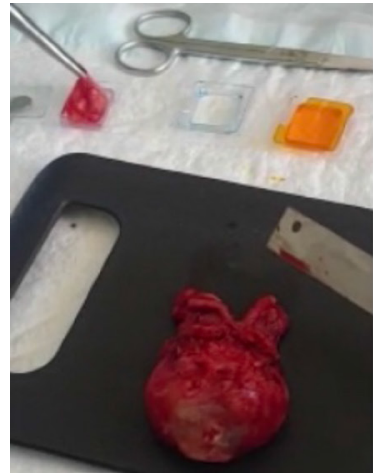

Preparation of a prostate apex; saline, and orange acridine staining for 30 seconds

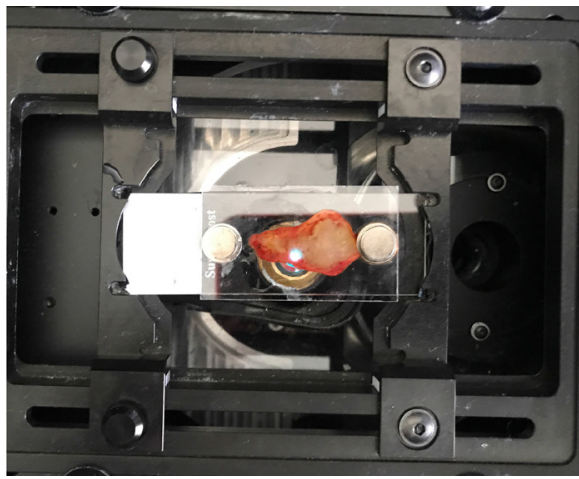

Fresh specimen (lateral margin) inside a glass, located in the microscope; scanning with laser from the bottom, the microscopical image appears on the main screen

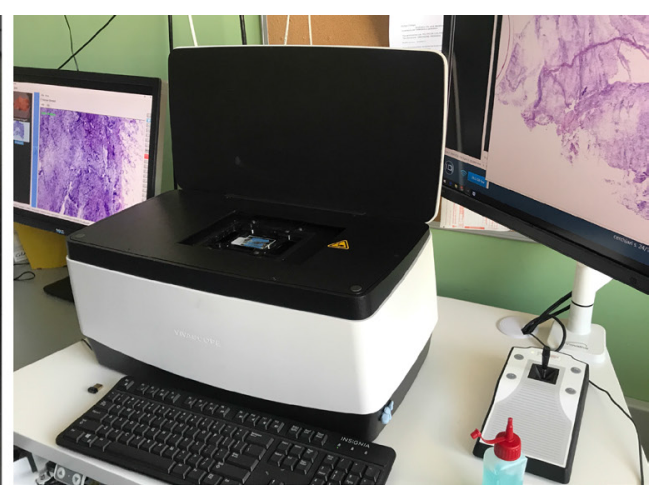

Setup of confocal microscope. From the right: main screen, a pc where the image instantaneously appears while scanning; the microscope, keyboard and mouse; additional screen

Figure S1 Confocal microscope setup. 